\title{
ZUR NUMERISCHEN LÖSUNG VON RANDWERTAUFGABEN BEI GEWÖHNLICHEN DIFFERENTIALGLEICHUNGEN.
}

VoN

\author{
E. J. NYSTRÖM \\ in HeLsingrors.
}

\section{§. Die Randwertanfgabe.}

1. Im folgenden betrachten wir Differentialgleichungen zweiter Ordnung

$$
\frac{d^{2} y}{d x^{2}}=f(x, y)
$$

in denen die erste Ableitung fehlt, insbesondere lineare Differentialgleichungen zweiter Ordnung

$(2)$

$$
\frac{d^{2} y}{d x^{8}}=g(x) y+h(x)
$$

wobei $f(x, y)$, bzw. $g(x)$ und $h(x)$ gegebene Funktionen sind.

Da die Integration, auch bei der Gleichung (2), nur durch Spezialisierung der rechten Seite weiter geführt werden kann, in den Anwendungen aber beliebige, auch empirisch gegebene Funktionen $f$, bzw. $g$ und $h$ auftreten, sind Näherungsmethoden zur Bestimmung der jeweils gesuchten Integralkurven von Bedeutung.

Da die allgemeine Lösung von (I) bzw. (2) zwei Integrationskonstanten enthält, kann man für eine einzelne Integralkurve zwei Bedingungen aufstellen. Während die Anfangswertaufgabe, d. h. die Ermittelung der durch einen gegebenen Punkt in gegebener Richtung laufenden Integralkurve, ziemlich erschöpfend untersucht worden ist, und man wohl in allen praktisch vorkommenden Fällen befriedigende Resultate erzielen kann, sind Randwcrtaufgalen verhältnismässig wenig untersucht worden, bei denen die Integralkurve Bedingungen an zwei verschiedenen Stellen zu erfüllen hat, obgleich in den Anwendungen gerade solche Probleme von grosser Bedeutung sind.

1-632047 Actu mathematica. 76:3-4 
2. Wir behandeln eingehend die sog. Randwertaufgabe erster Art, die Bestimmung der durch zwei gegebene Punkte $A\left(x=x_{a}, y=y_{a}\right)$ und $B\left(x=x_{b}, y=y_{b}\right)$ gehenden Integralkurve. Für andere Randwertaufgaben können im Prinzip ähnliche Formelsysteme aufgestellt werden, wie die von uns gegebenen (vgl. Nr. 22).

Man kann durchaus nicht behaupten, dass die in exakter Behandlung schwierigere Randwertanfgabe auch vom Standpunkt der praktischen Mathematik die schwierigere sei. Es lässt sich vielmehr denken, dass die Integralkurve durch zwei Punkte in einem gewissen Abstand leichter und genauer bestimmt werden kann, als die durch ein Linienelement, d. h. durch zwei zusammenfallende Punkte gehende.

Die gegebenen Funktionen $f$, bzw. $g$ und $h$ wollen wir zunächst für $x_{a} \leqq x \leqq x_{b}$ stetig und beschränkt annehmen; ferner setzen wir die Existenz einer stetigen Lösung des zu betrachtenden Randwertproblems voraus. ${ }^{1}$

3. Im folgenden. wird ein rom Verf. bereits im Jabre 1929 angegebenes Verfahren" zur Lösung der charakterisierten Randwertanfgabe ausführlich besprochen; verschiedene Vereinfachnngen, Verbesserungen und Erweiterungen werden hier gegeben sowie neue Beispiele angeführt.

Die zu leistende Rechenarbeit besteht, ausser eventuellen vorbereitenden Transformationen and der Ermittelung der Werte der gegebenen Funktionen in einzelnen Punkten, in der Lösung eines, im Falle der Gleichung (2) linearen Gleichungssystems mit zwei bis vier Unbekannten, von dessen Koeffizienten ein grosser Teil feste, d. h. von der Form der gegebenen Gleichung unabhängige Werte hat.

Eine Zusammenstellung der Formeln findet man am Schlusse der Arbeit.

\section{§ 2. Prinzipielle Losung.}

4. Für die Herleitung unserer Methode ist die Zurückführung der Randwertaufgabe auf ein bestimmtes Intervall und zwar auf die Strecke zwischen den Punkten $-\frac{1}{2}$ und $+\frac{1}{2}$ der Abszissenachse wesentlich. Wenn die Punkte $A\left(x_{a}, y_{a}\right)$

1 Utber die Existenz von Lösungen siehe KAMkE: Differentialgleichungen, Leipzig 1943, Bd. I, S. 279 .

'Soc. Scient. Fenn., Comm. Phys.-Math. V. 5 (1929), S. 17-21, ebenda XI. 4 (I942) nnd Acta mathematica 54 (1930), S. 185-204. Die Bezeichnang ist hier z. T. geăndert. 
Zur numerischen Lösung von Randwertaufgaben bei Differentialgleichungen. 159 und $\boldsymbol{B}\left(x_{b}, y_{b}\right)$ der Integralkurve gegeben sind, wenden wir zu dem gensnuten Zweck folgende Koordinatentransformation an

oder

$$
x=x_{a}\left(\frac{1}{2}-s\right)+x_{b}\left(\frac{1}{1}+s\right), \quad y=y_{a}\left(\frac{1}{2}-s\right)+y_{b}\left(\frac{1}{1}+s\right)+z
$$

$$
x=\left(x_{b}-x_{a}\right) s+\frac{1}{2}\left(x_{a}+x_{b}\right) \quad y=z+\left(y_{b}-y_{a}\right) s+\frac{1}{8}\left(y_{a}+y_{b}\right)
$$

Den Punkten $A$ und $B$ entsprechen nun die Pankte $\left(s=-\frac{1}{2}, z=0\right)$ und $\left(s=+\frac{1}{2}\right.$, $z=$ o). Ferner gilt

$$
\frac{d y}{d x}=\frac{d y}{d s}:\left(x_{b}-x_{a}\right), \quad \frac{d^{2} y}{d x^{2}}=\frac{d^{2} y}{d s^{2}}:\left(x_{b}-x_{a}\right)^{2}=\frac{d^{2} z}{d s^{2}}:\left(x_{b}-x_{a}\right)^{2} .
$$

Hierdurch entsteht die Randwertaufgabe

$$
\frac{d^{2} z}{d s^{2}}=\left(x_{b}-x_{a}\right)^{2} f(x, y), \quad z\left( \pm \frac{1}{2}\right)=0
$$

wo $x$ und $y$ gemäss (3) als Funktionen $x=x(s)$ and $y=y(s, z)$ von $s$ und $z$ ein. gesetzt werden sollen, also

$$
\frac{d^{2} z}{d s^{2}}=\left(x_{b}-x_{a}\right)^{2} f(x(s), y(s, z)), \quad z\left( \pm \frac{1}{2}\right)=0
$$

Denkt man sich die Funktion $z=\varepsilon(8)$ bekannt, so kann die rechte Seite von (5) als eine Funktion $\vec{f}(s)$ von $s$ betrachtet werden.

Im Falle der Gleichung (2) erhält man durch die Transformation (3)

$$
\frac{d^{2} z}{d s^{2}}=\bar{g}(s) z+h(s), \quad z\left( \pm \frac{1}{2}\right)=0
$$

wo die neuen Koeffizienten $\bar{g}$ und $\hbar$ sind:

(7) $\left\{\begin{array}{l}\bar{g}(s)=\left(x_{b}-x_{a}\right)^{2} g(x(s)) \\ h(s)=\left(x_{b}-x_{a}\right)^{2} g(x(s))\left[\left(y_{b}-y_{a}\right) s+\frac{1}{2}\left(y_{a}+y_{b}\right)\right]+\left(x_{b}-x_{a}\right)^{2} h(x(s))\end{array}\right.$

5. Die Ermittelung einer Lösung der Randwertaufgabe (5), bzw. (6) ist äquivalent damit, eine gewisse Integralgleichung zu lösen ${ }^{1}$. Sie lautet, wenn wir $t$ als

I Dieser Zusammenhang dürfte zuerst von BurkrardT (Bulletin de la Bociété mathématiqne de France, 1894) erkannt worden sein. 
Integrationsvariable einführen und daher in dem Ausdruck $f(x(s), y(s, z)) s$ durch $t$ ersetzen,

$$
z(s)=\left(x_{b}-x_{a}\right)^{2} \int_{-\frac{1}{2}}^{+\frac{1}{2}} K(s, t) f(x(t), y(t, z(t))) d t
$$

wo der $\operatorname{Kern} K(s, t)$ folgendermassen definiert ist:

(9)

$$
\begin{aligned}
& K(s, t)=\left(\frac{1}{2}-s\right)\left(\frac{1}{2}+t\right) \quad \text { für } \quad-\frac{1}{2} \leqq t \leqq s \\
& K(s, t)=\left(\frac{1}{2}+s\right)\left(\frac{1}{2}-t\right) \quad \text { für } \quad s \leqq t \leqq+\frac{1}{2}
\end{aligned}
$$

Der Kern $K(s, t)$ selbst ist eine im Intervall $\left(-\frac{1}{2},+\frac{1}{2}\right)$ stetige Funktion: für $t=s$ hat man $K(s, s)=\frac{1}{4}-s^{2}$; jedoch ist seine Ableitung für $t=s$ immer unstetig. Um sich von der Richtigkeit der Integralgleichung (8) zu überzeugen, braucht man nur diese zweimal nach dem Parameter $s$ zu differenzieren, indem man die Integrale gemäss (9) und (9)' in zwei Teile zerlegt und beachtet, dass sowohl die Integranden wie die Grenzen von $s$ abhängen. Für $s=-\frac{1}{2}$ und $s=\frac{1}{2}$ findet man $z(s)=\mathrm{o}$.

6. Der linearen Randwertaufgabe (6) entspricht die lineare Integralgleichung

$$
z(s)=\int_{-\frac{1}{2}}^{+\frac{1}{2}} K(s, t) \bar{g}(t) z(t) d t+\int_{-\frac{1}{2}}^{+\frac{1}{2}} K(s, t) h(t) d t
$$

wo der Kern wieder durch (9), (9)' definiert ist.

Man kann die Lösung der Randwertaufgabe (5), bzw. (6) als durch die Formeln (8), bzw. (Io) definiert ansehen, wenn das von der unbekannten Funktion abhängige Integral der rechten Seite durch einen mit genügender Genauigkeit approximierenden Ausdruck ersetzt wird. Aus der Integralgleichung werden wir die bereits in Nr. 3 angedenteten Gleichungssysteme ableiten. Im folgenden Paragraphen werden wir Mittel besprechen, welche die betreffende Approximation auszuführen gestatten.

\section{§ 3. Anwendung von Mittelwertmethoden.}

7. Zwecks Approximation des Integrals in (8) kommen sog. Mitteluertmethoden in Betracht, wobei die Integrale durch zweckmässig gewählte Summen ersetzt werden. Solche Approximationen lassen sich auch dann ausführen, wenn der Integrand, wie hier, von einem Parameter $s$ abhängt. 
Zur numerischen Lösung von Randwertaufgaben bei Differentialgleichungen. 161

Bei den betreffenden Methoden wird ein "gewogenes Mittel» von dem Wert des Integranden in einigen, wenigen Punkten des Integrationsintervalles gebildet. Man kann die Wahl der Punkte und der Gewichtszahlen mit Rücksicht auf die Bequemlichkeit der Rechnung rornehmen oder aber mit Rücksicht auf die Genauigkeit des Resultats. Ferner kann man vorschreiben, dass die Werte des Integranden in gewissen Punkten mitgenommen werden sollen.

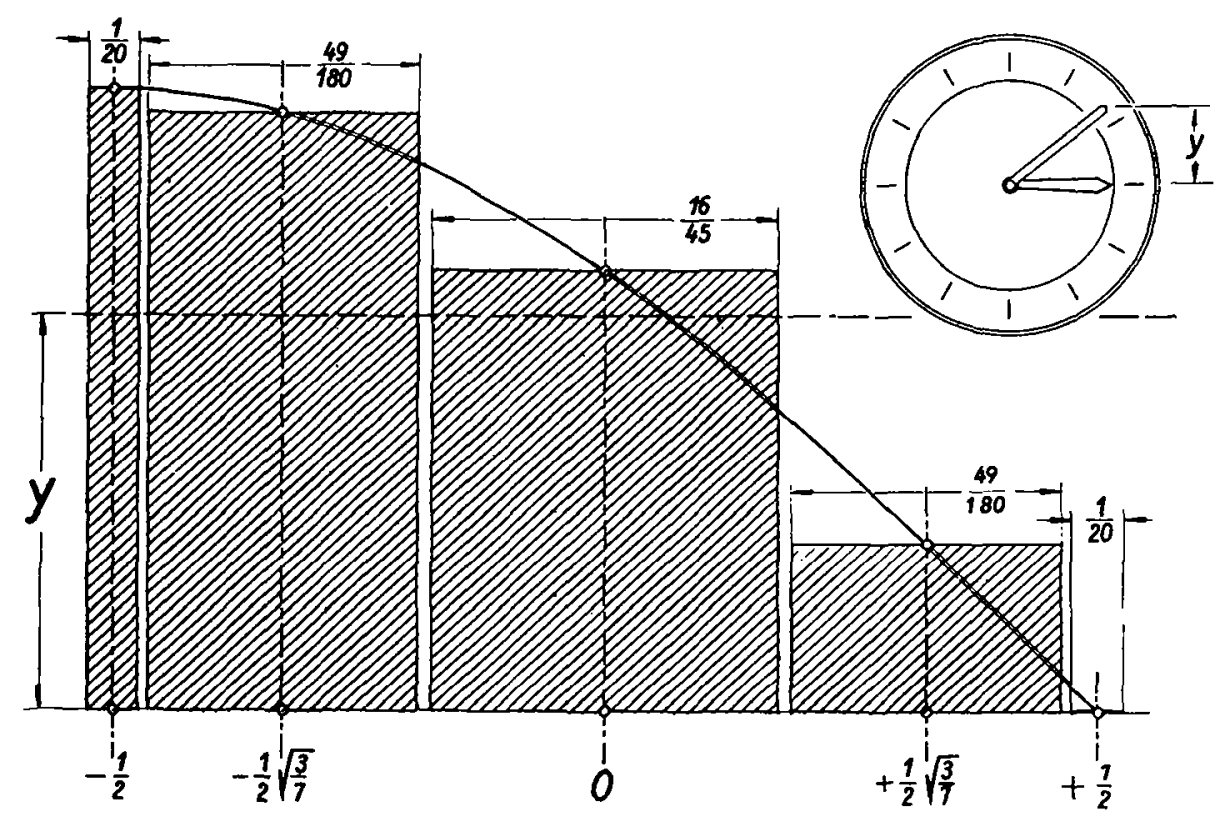

Abb. I.

Solche Methoden liefern bei Anwendung auf genügend regelmässig verlaufende Funktionen eine fast erstaunliche Genauigkeit. Wir geben ein Beispiel:

Es wird nach der mittleren Höhe $y(t)$ der Spitze des Minutenzeigers einer Wanduhr als Funktion der Zeit während einer ersten Viertelstunde gefragt (Abb. I).

Wir wenden die sog. Gauss-Lobattosche Formel

$$
\int_{-\frac{1}{2}}^{+\frac{1}{9}} y(t) d t \approx \frac{1}{90} y\left(-\frac{1}{2}\right)+\frac{49}{180} y\left(-\frac{1}{2} \sqrt{\frac{y}{y}}\right)+\frac{16}{13} y(0)+\frac{49}{180} y\left(+\frac{1}{2} \sqrt{\frac{3}{7}}\right)+\frac{1}{20} y\left(+\frac{1}{2}\right)
$$

an, welche das bestmögliche Resultat liefert, wenn fünf Ordinaten berücksichtigt werden und die Randordinaten sich unter diesen befinden sollen. Das Resultat wird überaus genau, der Fehler beträgt weniger als ein Millionstel der Uhrzeigerlänge. 
In Abb. I ist die Gauss-Lobattosche Formel versnschanlicht. Die Lage and Breite der Rechtecke entspricht den'zu benutzenden Ordinaten und deren Gewichtszahlen; sie sind also bis auf ihre Höhe unabhängig vom Verlanf der zu integrierenden Funktion innerhalb des festen Intervalls. Die Summe ihrer Flächeninhalte gibt mit grosser Genauigkeit den Flächeninhalt der von der Kurve und der $t$-Achse im Integrationsintervall begrenzten Figur wieder.

8. Mittelwertformeln zur angenäherten Integration, etwa

$$
\int_{t_{a}}^{t_{b}} y(t) d t \approx\left(t_{b}-t_{a}\right)\left(G_{a} y_{a}+G_{1} y_{1}+\cdots+G_{n} y_{n}\right)
$$

werden gefunden, indem man sowohl das Integral wie die endliche Summe in eine Taylorsche Reihe entwickelt und die Gewichtszahlen $G_{i}$ bzw. die Lage der Ordinaten $y_{i}$ in bestimmten Punkten $t=s_{i}(i=0, \mathrm{I}, 2, \ldots, n)$ so wählt, dass die Reihen in möglichst vielen Gliedern übereinstimmen. Es muss vorausgesetzt werden, dass die betreffende Taylorsche Entwicklung im ganzen Integrationsintervall $\left(t_{a}, t_{b}\right)$ gültig ist und gut konvergiert. In diesem Falle genügt eine geringe Anzahl Ordinaten, um den Verlauf der Funktion festzulegen.

9. Ziehen wir zur Approximation des Integrals in (8) eine Mittelwertformel heran, so tritt die unbekannte Funktion $z$ zunächst auf beiden Seiten des Gleichheitszeichens auf. Werden in der Mittelwertformel die Ordinaten in den Punkten $t=s_{i}$ benutzt, und setzt man in die Gleichung nacheinander die Werte $s=s_{i}$ ein, so erhält man ein Gleichungssystem zar Bestimmung der Werte $z_{i}$ der gesuchten Funktion $z(s)$.

Es erweist sich aber bequemer, die Werte $y_{i}$ der ursprünglich gesuchten Funktion $y(x)$ durch das Gleichungssystem direkt zu bestimmen. Zu diesem Zweck führen wir in (8) gemäss (3) die Funktion $y(s)$ wieder ein und schreiben also

$$
y-\left(y_{b}-y_{a}\right) s-\frac{1}{2}\left(y_{a}+y_{b}\right)=\left(x_{b}-x_{a}\right)^{2} \int_{-\frac{1}{2}}^{+\frac{1}{2}} K(s, t) f(x(t), y(t)) d t
$$

Die Einsetzung der Werte $s_{i}$ ergibt ein Gleichungssystem zur Bestimmung der gesuchten Grössen $y_{i}$.

10. Die Punkte $s_{i}$ werden zweckmässig im Intervall symmetrisch zur Mitte verteilt, wodurch gewisse Bedingungsgleichungen ohne weiteres erfüllt werden. Die erhaltenen Systeme zeigen dann eine gewisse Symmetrie. 
Zur numerischen Lösung von Randwertaufgaben bei Differentialgleichungen. 163

Infolge des stets vorhandenen Faktors $K(s, t)$ ist es zweckmässig, spezielle Mittelwertformeln für die uns interessierenden Integrale aufzustellen. Wir werden uns also nicht etwa der rorhin erwähnten Gauss-Lobattoschen Formel bedienen (vgl. Nr. I8).

Da wir die Integration nach $t$ stets ron $-\frac{1}{2}$ bis $+\frac{1}{2}$ ausfübren, setzen wir in (11) $s_{0}=-\frac{1}{2}, s_{n}=\frac{1}{2}$; ferner sind $y_{0}=y_{a}$ und $y_{n}=y_{b}$ die gegebenen Randwerte; $z_{a}$ und $z_{b}$ sind gleich Null.

Die sich ergebenden Gleichnngssysteme führen wir am Schlusse der Arbeit an und zwar für die Randwertaufgabe erster Art bei der allgemeinen Gleichung ( $I$ ). Darin treten Werte $y_{i}$ der gesuchten Funktion, "Zwischenordinaten", als Unbekannte auf. In dem wichtigen Spezialfall der linearen Gleichung (2) hat man einfach $f(x, y)$ durch $g(x) y+h(x)$ zu ersetzen, die Gleichungssysteme werden dann, wie gesagt, linear. Die von der Funktion $h(x)$ abhängigen Glieder sind durch die jeweils benutzte Mittelwertformel zu berechnen, wenn deren exakte Berechnung nicht durchführbar oder za umständlich ist.

\section{§ 4. Herleitang der Formeln.}

I1. Un geeignete Formeln zur Approximation eines Integrals der Form

$$
\int_{-\frac{1}{2}}^{+\frac{1}{2}} K(s, t) \vec{f}(t) d t
$$

zu gewinnen, gehen wir von der Taylorschen Entwicklung der Funktion $\bar{f}(t)$ im Mittelpunkt $t=0$ des Integrationsintervalles aus, welche nebst Restglied folgendermassen lautet

$$
\begin{gathered}
\bar{f}(t)=c_{0}+c_{1} t+c_{2} t^{2}+\cdots+c_{k} t^{k}+\frac{\overline{f^{(k+1)}(\xi)}}{(k+1) !} t^{k+1}, \\
(0<\xi<t),
\end{gathered}
$$

wobei $c_{0}=\bar{f}(\mathrm{o})$ und

$$
c_{i}=\frac{\bar{f}^{(i)}(o)}{i !} \quad(i=\mathrm{I}, 2, \ldots, k)
$$

Wir setzen, wie in $\mathrm{Nr} .8$ gesagt, die Gültigkeit dieser Entwicklung im ganzen Integrationsintervall $\left(-\frac{1}{2},+\frac{1}{2}\right)$ voraus. Geht man mit dieser Entwicklung in das 
obige Integral ein, braucht man nur die Produkte $K(s, t) t^{i}$ zu integrieren und findet

$$
\left\{\begin{array}{l}
\int_{-\frac{1}{3}}^{+\frac{1}{2}} K(s, t) t^{2 v} d t=\frac{\mathrm{I}}{(2 v+\mathrm{I})(2 v+2)}\left(\left(\frac{\mathrm{I}}{2}\right)^{2 v+2}-s^{2 v+2}\right), \\
\int_{-\frac{1}{2}}^{+\frac{1}{2}} K(s, t) t^{2 v+1} d t=\frac{s}{(2 v+2)(2 v+3)}\left(\left(\frac{1}{2}\right)^{2 v+2}-s^{2 v+2}\right) .
\end{array} \quad(v=0, \mathrm{I}, \ldots)\right.
$$

Wir wollen andererseits einen Mittelwert aus einer Anzahl Werte des Integranden mit möglichst guter Approximation finden. Da wir aber stets bestimmte Werte $s$ und $t$ einsetzen wexden und der $\operatorname{Kern} K(s, t)$, der als Faktor im Integranden auftritt, jedesmal einen von $\bar{f}$ unabhängigen Wert annimmt, können wir diesen als in den betreffenden Gewichtszahlen $G_{i}$ enthalten denken und wir können daher ebensogut den Mittelwert der Funktion $\overline{f(t)}$ bilden. Die entstehenden Glieder mit den $c_{i}$ werden wir mit den entsprechenden, durch exakte Integration des Produkts $K(s, t) \bar{f}(t)$ sich ergebenden vergleichen.

12. Bei der Approximation des Integrals in (12) benutzen wir zunächst den Mittelwert aus vier, in den Rand- und zwei inneren Punkten $t_{0}=-1, t_{1}=-t, t_{2}=t$, $t_{3}=+\frac{1}{2}$ zu nehmenden Ordinaten mit den Gewichten $G_{0}, G_{1}, G_{9}, G_{3}$. Hierbei ist $t$ eine noch verfügbare Zahl. Wir erhalten mit Hilfe von (14) dureh Vergleich der entsprechenden Glieder

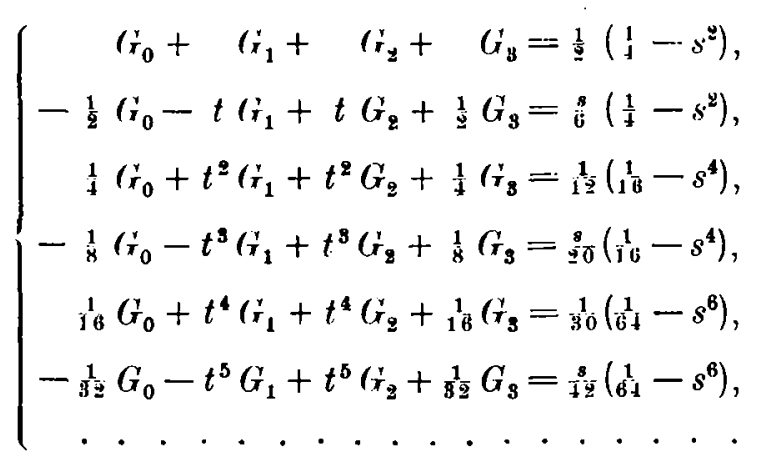

Wählen wir die vier Ordinaten äquidistant, setzen also $t=\frac{1}{6}$, d. h. $t_{1}=-\frac{1}{6}$, $t_{2}=+\frac{1}{6}$, so ergeben die vier ersten Gleichungen (15) völlig bestimmte Werte für die $G_{i}$ und zwar, wenn wir rechts zuerst $s=s_{1}=-\frac{1}{6}_{\text {einsetzen, }}$ einets

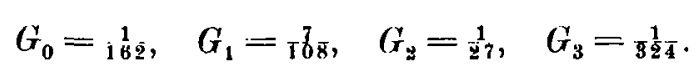


Zur numerischen Lösung von Randwertaufgaben bei Differentialgleichungen. 165

Mit dem Wert $s=s_{2}=+\frac{1}{6}$ ergeben sich aus (15) infolge der Symmetrie der Koeffizienten dieselben Werte $G_{i}$, aber in umgekehrter Reihenfolge. Die fünfte Gleichung in (15) wird nicht mehr befriedigt: man erhält auf der linken Seite 0,0006572 , während rechts 0,0005201 zu stehen kommt.

Aus (I2) erhalten wir also mit $s_{1}=-\frac{1}{6}$ und $s_{2}=+\frac{1}{6}$ zwei Gleichungen zur Berechnung der Werte $y_{1}$ und $y_{2}$ der gesuchten Funktion in den beiden Zwischenpunkten. Die betreffenden Formeln bilden die Gruppe II der Formelzusammenstellung, wo auch Korrektionsglieder angegeben sind. Wir nennen die Approximation eine solche $n$-ter Ordnung, wenn sie für alle Polynome vom höchstens $n$-ten Grade exakt ist. Die Hinzufügung der sersten Korrektionsglieder zu den er haltenen $y_{i}$ würde die Ordnung der Approximation um I erhöhen, also einen verbesserten, wenn auch nicht exakten Wert ergeben. Die Korrektionsglieder dienen im allgemeinen nur zum Vergleich der verschiedenen Formelsysteme, da sie sich selten bequem berechnen lassen.

13. Die beiden Punkte $-t$ und $t+$, in denen die $\mathrm{Zwischenordinaten} \mathrm{ge-}$ nommen werden, können wir aber so wählen, dass auch die fünfte Gleichung des Systems (15) befriedigt wird. Um dies zu erreichen, setzen wir $t=s=s_{1}$ und eliminieren die Gewichte $G_{i}$ aus den fünf ersten Gleichungen, wodurch wir eine Gleichung für $s_{1}$ erhalten. Diese liefert eine einzige, brauchbare Wurzel

$$
s_{1}=-\sqrt{\frac{\mathrm{II}-\sqrt{\mathrm{II} 2}}{\mathrm{I} 2}}=-0,1864124=-s_{z} .
$$

Durch Finsetzen dieses Wertes lassen sich aus den vier ersten Gleichungen (I 5) die, jetzt irrationalen, Gewichtszahlen $G_{i}$ berechnen und ferner, aus der sechsten Gleichung, die ersten Korrektionsglieder.

Das neue Formelsystem, welches ja eine verschärfte Approximation liefert, ist III.

I 4. Werden zur Mittelwertbildung drei Zwischenordinaten herangezogen, sind diese wegen der Symmetrie folgendermassen zu wählen

$$
t_{1}=-t, \quad t_{8}=0, \quad t_{3}=t
$$

wobei $t$ zunächst noch unbestimmt sein möge. Dann wird das den Gleichungen (I 5) entsprechende System: 


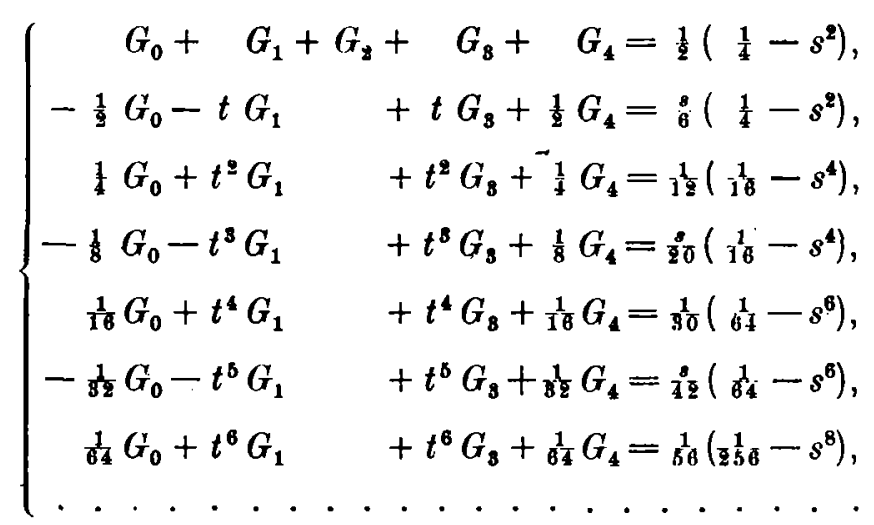

Wählen wir die Ordinaten äquidistant, setzen. also $t=\frac{1}{4}$, so ergeben die fünf ersten Gleichangen ( 6 ) völlig bestimmte Werte für die Gewichtszahlen $G_{i}$, nämlich mit $s_{1}=-\frac{1}{4}$ und mit $s_{3}=+\frac{1}{1}$ dieselben Werte, aber in umgekehrter Reihenfolge, ferner mit $s_{2}=0$ Werte, bei denen $G_{0}=G_{4}$ und $G_{1}=G_{3}$.

Durch Einsetzen der Werte $s_{1}=-1, s_{2}=0, s_{3}=+\frac{1}{4}$ in (12) bekommen wir also drei Gleichungen zur Bestimmung der Grössen $y_{1}, y_{2}, y_{3}$. Die sechste Gleichung in (16) gilt für $s_{1}$ und $s_{8}$ nicht mehr, wohl aber für $s_{2}$. Aus der 6. und der 7 . Gleichnng werden die ersten Korrektionsglieder berechnet. Die Genanigkeit des so erhaltenen Systems IV ist, wie aus den Korrektionsgliedern hervorgeht, grösser als die des. Systems III, wenn auch beide Systeme Approximationen 4. Ordnung geben.

15. Eine verschärfte Approximation gewinnen wir wieder durch besondere Wahl der äusseren von den drei $\mathrm{Zwischenordinaten;} \mathrm{die} \mathrm{mittlere} \mathrm{ist} \mathrm{wegen} \mathrm{der}$ Symmetrie bei $t=0$ zu nehmen.

Wenn wir $t=s=s_{1}$ setzen und aus den sechs ersten Gleichungen (16) die fünf $G_{i}$ eliminieren, erhalten wir eine Gleichung für $s_{1}$, die eine einzige brauchbare Wurzel liefert, nämlich

$$
s_{1}=-\sqrt{\frac{19-\sqrt{240}}{44}}=-0,2823630=-s_{3} .
$$

Benutzt man diesen Wert statt des in Nr. 14 angewandten, so erhält man das Formelsystem V, welches eine Approximation 5. Ordnung gibt.

16. Gehen wir einen Schritt weiter zu dem Fall von vier Zwischenordinaten, so sind diese bei äquidistanter Verteilung in den Punkten

$$
t_{1}=-0,3, \quad t_{2}=-0,1, \quad t_{3}=+0,1, \quad t_{4}=+0,3
$$


Zur numerischen Lösung von Randwertaufgaben bei Differentialgleichungen. 167 zu nehmen. Aus (13) und (14) erhalten wir folgende Gleichungen

$$
\begin{aligned}
G_{0}\left(-\frac{1}{2}\right)^{i}+G_{1}(-0,3)^{i}+\left(G_{2}(-0,1)^{i}+\right. & G_{3}(+0,1)^{i}+G_{4}(+0,3)^{i}+G_{3}\left(+\frac{1}{2}\right)^{i} \\
& =\int_{-\frac{1}{2}}^{+\frac{1}{2}} K(s, t) t^{i} d t \quad(i=0,1,2, \ldots) .
\end{aligned}
$$

Für jeden der Werte $s= \pm 0,3, s= \pm 0,1$ bekommen wir aus den sechs ersten dieser Gleichungen die betreffenden Gewichtszahlen $G_{i}$. Die letzteren sind ebenso wie bei den früher betrachteten äquidistanten Ordinatenverteilungen rational. Die siebente Gleichung liefert jeweils das erste Korrektionsglied. Das so gewonnene Formelsystem VI gibt eine Approximation 5. Ordnung und zeichnet sich durch besonders bequeme Koeffizienten aus.

Eine verschärfte Approximation lässt sich wohl ähnlich wie in den Nummern I 3 und I 5 herleiten, wir wollen aber darauf nicht eingehen.

17. Der Vollständigkeit wegen wollen wir untersuchen, was sich im Falle einer einzigen $\mathrm{Z}$ wischenordinate ergibt, die natürlich bei $t=0$ anzunehmen ist.

Wir können uns des Gleichungssystems (16) bedienen, wenn wir $G_{1}=G_{3}=0$ annehmen und die Indices 2 und 4 in 1 und 2 ändern. Die drei ersten Gleichungen werden, wenn rechts $s=0$ eingesetzt wird,

$$
\left\{\begin{aligned}
G_{0}+G_{1} & +G_{2}=\frac{1}{8} \\
-\frac{1}{2} G_{0}+\frac{1}{2} G_{2} & =0 \\
\frac{1}{4} G_{0}+\frac{1}{1} G_{2} & ={ }_{1}{ }_{2}
\end{aligned}\right.
$$

Hieraus folgt $G_{0}=G_{2}=\frac{1}{96}, G_{1}=\frac{5}{48}$. Diese Werte erfillen auch die vierte Gleichung und ergeben, in die fünfte eingesetzt, links $\frac{1}{768}$ statt $\frac{1}{1920}$. Für unsere Zusammenstellung erhalten wir also eine Formel I, die eine Approximation 3. Ordnung gibt.

\section{$\S$ 5. Genauigkeitsbetrachtungen. Interpolation.}

18. Die von uns in den Nummern II bis I 7 hergeleiteten Formelsysteme I bis VI sind speziell für die betrachtete Randwertaufgabe zageschnitten. Die Gauss-Lobattosche Formel (Nr. 8) z. B. ist zwar für einfache Quadraturen ausgezeichnet - sie gibt bei solchen eine Approximation 7. Ordnung --, jedoch 
ist die darin angewandte Ordinatenverteilung für unseren Zweck nicht sehr geeignet ${ }^{1}$.

Ist bei einer Approximation $n$-ter Ordnung die Funktion $\bar{f}$ in Nr. 4 ein Polynom vom $n$-ten Grade in $t$, so sind die berechneten Ordinaten exakt. Zur eindeutigen Bestimmung eines solchen Polynoms müssen nun seine Werte in $n+$ I Punkten bekannt sein. Wenn also die Anzahl der bekannten Ordinaten die 2 gegebenen Randwerte mitgezählt - wenigstens $n+$ I beträgt, so wird durch dieselben das Polynom $n$-ten Grades völlig bestimmt sein. In Tafel $I$ ist die jeweilige Anzahl der bekannten Ordinaten angegeben, ebenso der Höchstgrad der eventuel vorhandenen Polynomlösung, die durch unsere Approximation gefunden wird.

Ein Vergleich zeigt, dass nach diesem Gesichtspunkt die Formeln I, II, IV, VI, d. h. die mit äquidistanter Ordinatenverteilung den anderen, nämlich III, bzw. V vorzuziehen sind, weil sie die betreffenden exakten Polynomlösungen etwas bequemer finden lassen.

Richtiger ist es jedoch, die Beurteilung der Formeln auf die angegebenen Korrektionsglieder zu gründen.

19. Bevor wir an Erweiterungen und eigentliche Beispiele herangehen, wollen wir unsere Formeln an dem einfachen Fall erproben, wo in ( 13 ) alle $c_{i}$ gleich I sind. Es handelt sich dann darum, die Randwertaufgabe

$$
y^{\prime \prime}(x)=\frac{\mathrm{I}}{\mathrm{I}-x}=\mathrm{I}+x+x^{2}+\cdots, \quad y\left( \pm \frac{1}{2}\right)=\mathrm{o}
$$

zu lösen, welche übrigens als die Bestimmung der elastischen Linie eines gewissen Trägers auf zwei Stützen aufgefasst werden kann. Die exakte Lösung ist $y=(\mathrm{r}-x) \log \operatorname{nat}(\mathrm{r}-x)+0,9547712 x-0,1308$ 1 20, Abb. 2.

1 Das vom Verf. früher gebranchte, aus der Gauss-Labattoschen Formel hergeleitete Formelsystem ist also nicht besonders empfehlenswert. Die Korrektionsglieder dieses Formelsystems

$$
\Delta y_{1}=-\Delta y_{3}=+0,0000227 \cdot c_{5}, \quad \Delta y_{2}=-0,0000233 \cdot c_{6}
$$

sind in der Tat grösser als die entsprechenden des Systems IV. In der Arbeit Soc. Scient. Fenn., Comm. Phys.-Math. XI. I4, S. 14 sind übrigens in dem Ausdruck für $-f_{2}$, die Vorzeichen von $\bar{\beta}_{0}$ und $\bar{\beta}_{4}$ in - zu ändern. Die Arbeit des Verf. in Acta mathematica 54 (1930) entbält auch ein Formelsystem, nämlich (24), S. 202 mit Gauss-Lobattoscher Ordinatenverteilung. Dasselbe muss ebenfalls berichtigt werden: Es gilt nämlich nur, wenn, wie in dem darauf folgenden Beispiel, $F\left(-\frac{1}{2}, 0\right)=F\left(+\frac{1}{2}, 0\right)=0$. Sonst sind die betreffenden Glieder hinzuzufügen, wie sie sich aus der Gauss-Lobattoschen Formel ergeben, nämlich für die erste Gleichung -0,0005208 $\left(F\left(-\frac{1}{2}, 0\right)+F\left(+\frac{1}{2}, 0\right)\right)$, für die zweite und dritte Gleichung $+0,0003400\left(F\left(-\frac{1}{2}, 0\right)+F\left(+\frac{1}{2}, 0\right)\right)$. 
Zur numerischen Lösung von Randwertaufgaben bei Differentialgleichungen. 169

Die Ergebnisse der Anwendung unserer Formeln sind in Tafel I zusammengestellt. Auf das Frgebnis der Formel I kommen wir später (Nr. 2I) zu sprechen; die Gruppen II bis VI liefern Werte $y_{i}$, welche in zeichnerischer Darstellung nicht merklich von den exakten abweichen.

Tafel $I$.

\begin{tabular}{|c|c|c|c|c|c|c|c|c|c|c|c|c|c|c|c|}
\hline Formelsystem & I & \multicolumn{2}{|c|}{ II } & \multicolumn{2}{|c|}{ III } & \multicolumn{3}{|c|}{ IV } & \multicolumn{3}{|c|}{ V } & \multicolumn{4}{|c|}{ VI } \\
\hline Anzahl der Ordinaten & 3 & \multicolumn{2}{|c|}{4} & \multicolumn{2}{|c|}{4} & \multicolumn{3}{|c|}{5} & \multicolumn{3}{|c|}{5} & \multicolumn{4}{|c|}{6} \\
\hline $\begin{array}{l}\text { Ordnung der } \\
\text { Approximation }\end{array}$ & 3 & \multicolumn{2}{|c|}{3} & \multicolumn{2}{|c|}{4} & \multicolumn{3}{|c|}{4} & \multicolumn{3}{|c|}{5} & \multicolumn{4}{|c|}{5} \\
\hline $\begin{array}{l}\text { Höchstgrad der } \\
\text { Polynomlösung }\end{array}$ & 2 & \multicolumn{2}{|c|}{3} & \multicolumn{2}{|c|}{3} & \multicolumn{3}{|c|}{4} & \multicolumn{3}{|c|}{4} & \multicolumn{4}{|c|}{5} \\
\hline$i$ & I & $\mathbf{I}$ & 2 & $\mathbf{I}$ & 2 & $\mathbf{I}$ & 2 & 3 & I & 2 & 3 & I & 2 & 3 & 4 \\
\hline 8 & o & $-\frac{1}{11}$ & $+\frac{1}{6}$ & $\begin{array}{l}-s \\
(s=0\end{array}$ & $\begin{array}{c}+s \\
\left(86_{4} r\right.\end{array}$ & $-\frac{1}{4}$ & 0 & $+\frac{1}{4}$ & $\begin{array}{l}-s \\
(s=\end{array}$ & $\begin{array}{c}0 ! \\
0,28\end{array}$ & $\begin{array}{l}+8 \\
2363\end{array}$ & $-0,3$ & $-0, I$ & $-+0,1$ & $+0,3$ \\
\hline $\begin{array}{c}\text { Beispiel: } \\
y^{\prime \prime}=\frac{\mathrm{I}}{\mathrm{I}-x}, y\left( \pm \frac{1}{2}\right)=0 \\
\text { Exakter Wert } y_{i}\end{array}$ & $\begin{array}{c}\infty \\
0 \\
0 \\
0 \\
0 \\
0\end{array}$ & $\begin{array}{l}0 \\
5 \\
0 \\
0\end{array}$ & 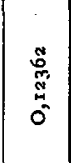 & $\begin{array}{l}: \\
: \\
\vdots \\
0 \\
0\end{array}$ & 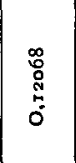 & 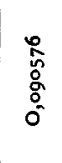 & $\begin{array}{l}\stackrel{0}{0} \\
\stackrel{0}{0} \\
\stackrel{m}{0} \\
0\end{array}$ & 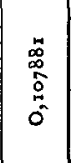 & $\begin{array}{c}\frac{1}{9} \\
4 \\
0 \\
0 \\
0 \\
0\end{array}$ & $\begin{array}{c}N \\
\text { s. } \\
0 \\
0 \\
0 \\
0\end{array}$ & 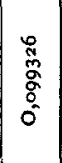 & \begin{tabular}{l}
0 \\
\multirow{0}{0}{} \\
0 \\
0 \\
0 \\
0
\end{tabular} & \begin{tabular}{c}
$\infty$ \\
\multirow{+}{+}{} \\
$\stackrel{0}{N}$ \\
0 \\
0
\end{tabular} & $\begin{array}{l}\stackrel{0}{0} \\
\stackrel{0}{0} \\
\stackrel{0}{0} \\
0\end{array}$ & $\begin{array}{l}3 \\
3 \\
0 \\
0 \\
0 \\
0 \\
0\end{array}$ \\
\hline $\begin{array}{c}\text { Approximativer } \\
\text { Wert } y_{i}\end{array}$ & $\begin{array}{l}0 \\
0 \\
0 \\
0 \\
0\end{array}$ & 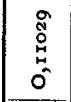 & 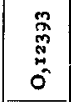 & $\begin{array}{l}\stackrel{2}{2} \\
0 \\
0 \\
0 \\
0\end{array}$ & $\begin{array}{c}0 \\
\hat{N} \\
\underline{0} \\
0\end{array}$ & $\begin{array}{l}m \\
0 \\
0 \\
8 \\
0 \\
0 \\
0\end{array}$ & $\begin{array}{l}m \\
\infty \\
0 \\
0 \\
0 \\
0\end{array}$ & $\begin{array}{l}\frac{+}{2} \\
\hat{\sigma} \\
0 \\
0\end{array}$ & 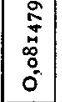 & $\begin{array}{l}\text { N } \\
\text { on } \\
0 \\
0 \\
0 \\
0 \\
0\end{array}$ & $\begin{array}{l}\text { o } \\
0 \\
0 \\
0 \\
0 \\
0\end{array}$ & $\begin{array}{l}0 \\
0 \\
0 \\
0 \\
0 \\
0 \\
0\end{array}$ & 尔 & $\begin{array}{l}\stackrel{0}{0} \\
\stackrel{0}{0} \\
0 \\
0\end{array}$ & $\begin{array}{l}0 \\
0 \\
\vdots \\
\vdots \\
0 \\
0 \\
0\end{array}$ \\
\hline $\begin{array}{l}\text { Korrektion in Einheiten } \\
\text { der letaten Stelle }\end{array}$ & - I I & --19 & -31 & +1 & $-\mathbf{I} \mathbf{I}$ & +3 & $-2 I$ & -53 & -4 & o & -13 & -8 & -9 & -10 & -15 \\
\hline $\begin{array}{l}\text { Abgeschätzter } \\
\text { Fehlerbetrag }\end{array}$ & -16 & -27 & -27 & +7 & -7 & +30 & -23 & -30 & -8 & 4 & -8 & $-\mathrm{I} 2$ & -10 & -10 & $-\mathrm{I} 2$ \\
\hline
\end{tabular}

20. Um rechnerisch Werte der gesuchten Funktion $y$ in beliebig gewählten Punkten zu ermitteln, muss man sich der Interpolation durch Polynome bedienen, da man im allgemeinen nur ihren Wert in 4, 5 oder 6 Punkten $s_{i}$ kennt. Die betreffenden Interpolationspolynome sind vom 3., 4. oder 5. Grade. Uns interessiert jetzt die Grösse des bei ihrer Anwendung entstandenen Fehlers, für den der allgemeine Ausdruck gilt

$$
\frac{\mathrm{I}}{(m+2) !}\left[\frac{d^{m+2} y}{d x^{m+2}}\right]_{x=\xi}\left(x^{2}-\frac{1}{4}\right)\left(x-s_{1}\right)\left(x-s_{2}\right) \ldots\left(x-s_{m}\right)
$$


wo $m$ die Anzahl der Zwischenordinaten ist und $\xi$ ein gewisser Wert im Intervall $\left(-\frac{1}{2},+\frac{1}{2}\right)$.

Aus (17) folgt für die Koeffizienten in (18)

$$
\frac{\mathrm{I}}{(m+2) !}\left[\frac{d^{m+2} y}{d x^{m+2}}\right]_{x=\xi}=\frac{\mathrm{I}}{(m+\mathrm{I})(m+2)(\mathrm{I}-\xi)^{m+1}} .
$$

II.

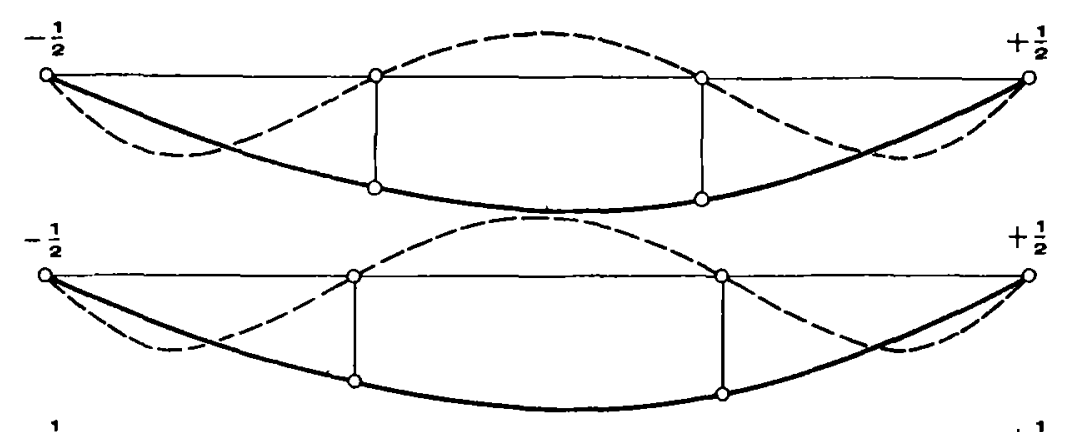

IV.

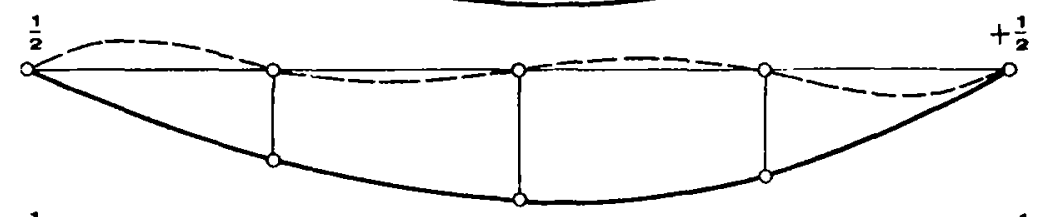

v.

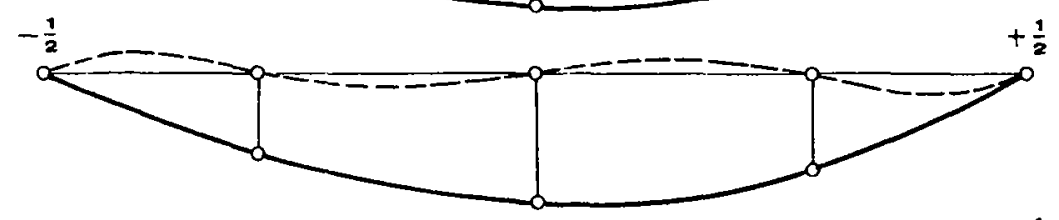

VI.

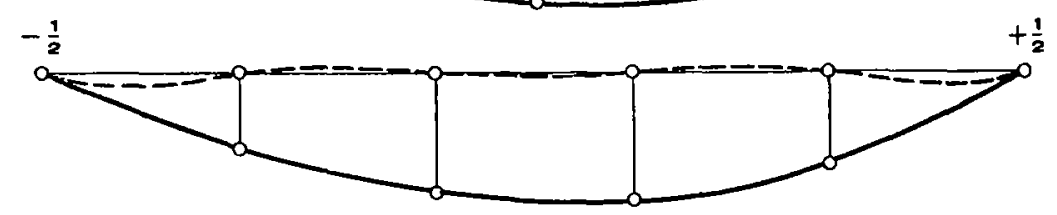

Abb. 2 .

Für $m=2,3,4$, wie je nach der Anzahl der Zwischenordinaten bei den Formelsystemen II, III, IV, V und VI zu setzen ist, erhalten wir mit $\xi=\frac{1}{2}$ als obere Grenzen $M_{n}$ der Grössen (19) die Werte $\frac{2}{3}, \frac{4}{5}, \frac{16}{16}$, welche in (18) einzusetzen sind. In $\mathrm{Abb} .2$ sehen wir die betreffenden fünf gestrichelten Kurven

$$
y=\operatorname{IOO} M_{m}\left(x^{2}-\frac{1}{4}\right)\left(x-s_{1}\right)\left(x-s_{z}\right) \ldots\left(x-s_{m}\right) .
$$

Die Interpolationsfehler erreichen an keiner Stelle das Hundertstel der Ordinaten der gestrichelten Kurven, sie werden im allgemeinen viel kleiner sein. 
Zur numerischen Iösung von Randwertaufgaben bei Differentialgleichungen. 171

Wie aus dem Gesagten hervorgeht, lassen sich die Werte der gesuchten Funktion im ganzen Intervall etwa dreistellig ermitteln; in den ausgewählten Punkten $s_{i}$ ist ja die Genauigkeit der $y_{i}$ mehrstellig.

Wenn auch unser erstes Beispiel ganz speziell ist, gibt es doch eine Vorstellung von dem, was mit den entwickelten Mitteln erreicht werden kann.

21. Im Falle des betrachteten Beispiels $y^{\prime \prime}(x)=\frac{1}{1-x}, y\left( \pm \frac{1}{2}\right)=0$ ergibt sich durch Anwendung unserer einfachsten Formel I $y(0)=0,1319$, welcher Wert um $0, \infty 11 \mathrm{zu}$ gross ist (vgl. Tafel I).

Da eine Interpolation zwischen den drei Werten $y\left( \pm \frac{1}{2}\right)$ und $y(0)$ kaum in Frage kommt, kann man zur Gewinnung anderer Werte der gesuchten Funktion $y(s)$ besser dieselbe Randwertmethode getrennt anf die beiden Intervallhälften von $-\frac{1}{2}$ bis $\circ$ und von $\circ$ bis $+\frac{1}{6}$ anwenden. Die nötigen Transformationen sind gemäss (6) und (7) leicht auszuführen, eine Aufstellung besonderer Formeln hierfür ist unnötig. Als Resultat wird gefunden

$$
\begin{aligned}
& \text { approximativ } \quad y\left(-\frac{1}{4}\right)=0,0911, \quad y\left(+\frac{1}{1}\right)=0,1085, \\
& \text { exakt } \quad y\left(-\frac{1}{1}\right)=0,0906, \quad y\left(+\frac{1}{1}\right)=0,1079 \text {. }
\end{aligned}
$$

Die Fehler dieser Grössen sind also o,0005 und o.0oo6, d. h. etwa die Hälfte des Fehlers von $y(0)$. Somit ist, wie zu erwarten, die Genauigkeit der zuerstberechneten mittleren Ordinate $y(0)$ für die nachher berechneten massgebend.

Ganz ungenau ist also das Ergebnis unserer einfachsten Methode nicht, in graphischer Darstellung würde es von demjenigen des Formelsystems IV in Abb. 2 nicht zu unterscheiden sein! Entsprechendes werden auch weitere Beispiele zeigen.

\section{§ 6. Lrweiterungen.}

22. In Nr. 2 ist als Randwertaufgabe erster Art die Aufgabe bezeichnet, eine Lösung $y(x)$ von (I) zu finden, welche für $x=x_{a}$ und $x=x_{b}$ vorgeschriebene Werte annimmt. Diese Werte können ohne Einschränkung als Null angenommen werden.

Als Randwertaufgabe zweiter Art gilt die, wo für $x=x_{a}$ und $x=x_{b}$ die Ableitung $y^{\prime}(x)$ vorgeschriebene Werte annehmen soll. 
Die Randwertaufgabe dritter Art ${ }^{2}$ enthält diejenigen erster und zweiter Art als Spezialfälle und schreibt der zu bestimmenden Funktion die Bedingungen vor

$$
\alpha^{\prime} y^{\prime}\left(x_{a}\right)+\alpha y\left(x_{a}\right)=\gamma, \quad \beta^{\prime} y^{\prime}\left(x_{b}\right)+\beta y\left(x_{b}\right)=\delta,
$$

wo $a, a^{\prime}, \beta, \beta^{\prime}, \gamma, \delta$ gegebene Konstanten sind.

Der Randwertanfgabe dritter Art für die Differentialgleichung (I), bzw. (2) entspricht die Integralgleichung (8), bzw. (10), wenn für den Kern ein geeigneter, von der speziellen Form der Funktionen $f, g, h$ unabhängiger Ausdruck

$$
\begin{array}{lll}
K(s, t)=A+B s+C^{\prime} t+D_{s} t & \text { für } & x_{a} \leqq t \leqq s \\
K(s, t)=A^{\prime}+B^{\prime} s+C^{\prime} t+D^{\prime} s t & \text { für } & s \leqq t \leqq x_{h}
\end{array}
$$

angenommen wird. Die Koeffizienten des Kerns hängen natürlich von den gegebenen Randbedingungen (20) ab.

Für bestimmte Formen der Randwertaufgabe dritter Art können nun Gleichungssysteme zur numerischen Approximation aufgestellt werden, wie wir es in $\S 4$ für diejenige erster Art getan haben. Für den allgemeinen Fall kommen solche Formeln nicht in Betracht, weil die Koeffizienten derselben von den Grössen $\alpha, \alpha^{\prime}, \ldots$ abhängig sein würden.

23. Unser Verfahren ist auch auf Randwertaufgaben bei Systemen von Differentialgleichungen zweiter Ordnung anwendbar, in denen die ersten Ableitungen feblen

$$
\left\{\begin{array}{l}
\frac{d^{2} x}{d t^{2}}=f\left(t, d^{\prime}, y\right) \\
\frac{d^{2} y}{d t^{2}}=g(t, x, y) .
\end{array}\right.
$$

Auf diese Form lässt sich jedes Normalsystem

$$
\left\{\begin{array}{l}
\frac{d x}{d t}=h(t, x, y), \\
\frac{d y}{d t}=-h(t, x, y)
\end{array}\right.
$$

bringen, wenn man nach der unabhängigen Veränderlichen $t$ deriviert und die ersten Ableitungen aus den gegebenen Gleichungen in die neuen einsetzt. Systeme der Form (22) kommen ja übrigens oft als solche vor, in der Mechanik

1 KAмкE, loc. cit. S. 255 . 
Zur numerischen Lösung von Randwertaufgaben bei Differentialgleichungen. 173

z. B., wenn die wirkenden Kräfte von dem Ort und der Zeit abhängen, nicht aber von den jeweiligen Geschwindigkeiten.

Die Randwertaufgabe möge darin bestehen, diejenigen Funktionen $x(t)$ und $y(t)$ ans (22) zu bestimmen, für welche die Bedingungen erfüllt sind:

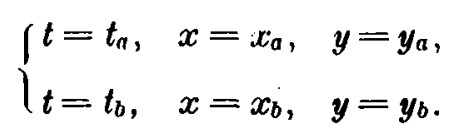

Um unsere Randwertaufgabe wieder auf das feste Intervall $\left(-\frac{1}{2},+\frac{1}{2}\right)$ zurückzuführen, setzen wir als Verallgemeinerung von (3)

$$
\left\{\begin{array}{l}
t=\left(t_{b}-t_{a}\right) s+\underline{\underline{1}}\left(t_{a}+t_{b}\right), \\
x=\bar{x}+\left(x_{b}-x_{a}\right) s+\frac{1}{2}\left(x_{a}+x_{b}\right), \\
y=\bar{y}+\left(y_{b}-y_{a}\right) s+\frac{1}{2}\left(y_{a}+y_{b}\right),
\end{array}\right.
$$

wobei $x$ und $y$ neue unbekannte Funktionen von $s$ sind. Ferner gilt

$$
\left\{\begin{array}{l}
\frac{d^{2} x}{d t^{2}}=\frac{d^{2} \bar{x}}{d s^{2}} \cdot \frac{\mathrm{I}}{\left(t_{b}-t_{a}\right)^{2}}, \\
\frac{d^{2} y}{d t^{2}}=\frac{d^{2} \bar{y}}{d s^{2}} \cdot \frac{1}{\left(t_{b}-t_{a}\right)^{2}} .
\end{array}\right.
$$

Unsere Formelsysteme I-VI können dann nach unmittelbar einzusehender Erweiterung angewandt werden.

Bei der Durchführung der Rechnung kann die in Nr. 9 angegebene divekte Bestimmung der Werte der gesuchten Funktion ohne vorherige Variabeltransformation erhebliche $\nabla$ orteile bringen.

Zur Erläuterung des in dieser Nummer Gesagten dient ein in Nr. 29 zu behandelndes, der Himmelsmechanik entnommenes Beispiel.

\section{§ 7. Beispiele.}

24. Beispiel 1. Zunächst betrachten wir die Differentialgleichung

$$
\frac{d^{2} y}{d x^{2}}=x^{2} y
$$

die nicht nur eine Beurteilung der Genanigkeit unserer Methode, sondern gewissermassen auch einen Vergleich mit der Anfangswertaufgabe gestattet. 
Für die Anfangsbedingungen $y(\mathrm{o})=\mathrm{I}, y^{\prime}(\mathrm{o})=\mathrm{o}$ lautet die Lösung dieser Gleichung

$$
y(x)=\mathrm{I}+\frac{x^{4}}{3 \cdot 4}+\frac{x^{8}}{3 \cdot 4 \cdot 7 \cdot 8}+\frac{x^{12}}{3 \cdot 4 \cdot 7 \cdot 8 \cdot \text { II } 12}+\cdots
$$

Es entsprechen einander folgende Werte von $x$ und $y^{1}$

$$
x_{i l}=\mathrm{o}, \quad y_{a}=\mathrm{I} ; \quad x_{b}=\mathrm{I}, 2, \quad y_{b}=\mathrm{I}, 1792999 .
$$

Wir wollen der Gleichung (25) diese Randbedingungen auferlegen (Abb. 3).

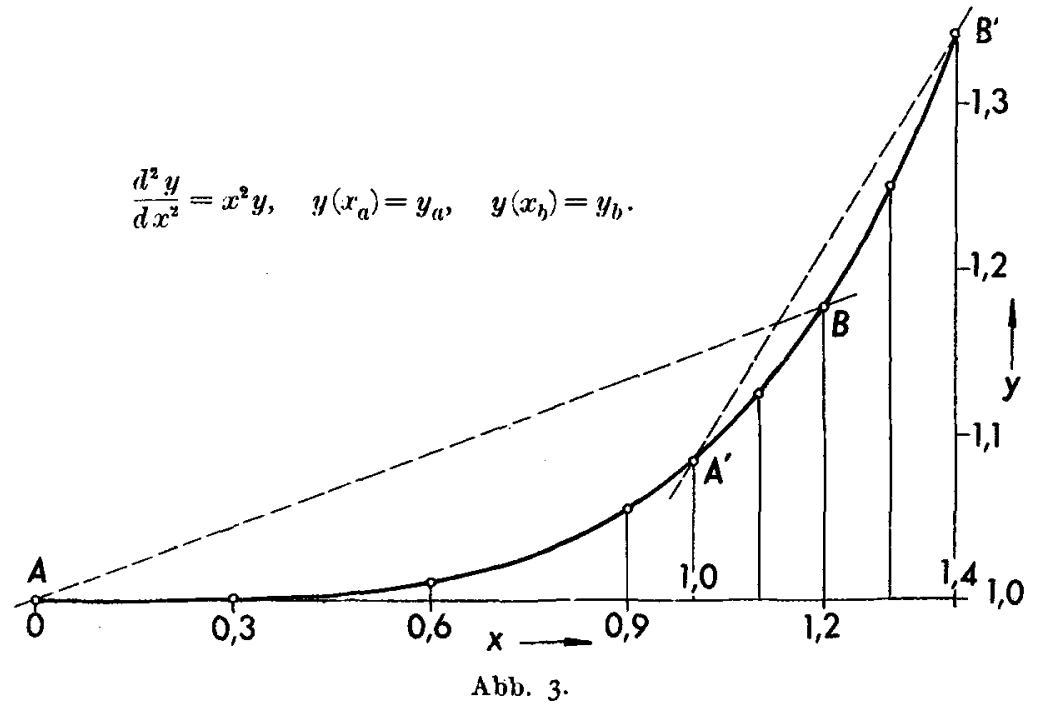

Zur unmittelbaren Bestimmung der drei Zwischenordinaten $y_{1}, y_{2}, y_{3}$ in den Punkten $x=0,3,0,6,0,9$ wenden wir das Formelsystem IV an. Wir brauchen hierbei nur statt $f(x, y)$ den Ausdruck $x^{2} y$ sowie die gegebenen numerischen Werte einzusetzen. Die entstehenden drei Gleichungen sind dann zu lösen, was durch Iteration leicht geschehen kann. Das Ergebnis geht aus Tafel II hervor und zeigt eine beträchtliche Genauigkeit.

Dieselbe Randwertaufgabe behandeln wir auch mit den Formelsystemen II und III, das Resultat ist in Tafel III angegeben. Wie zu erwarten war, liefert

1 Vgl. ERnst Lindedöf: Remarques sur l'intégration numérique des équations différentielles ordinaires (Acta Soc. Scient. Fenn., Nova Series A. II: I3), S. I 8 ff. Diese Arbeit enthält Verbesserungen bekannter Integrationsverfahren mit Hilfe von Differenzenschemata. Als Beispiel werden die Ordinaten der Kurve $y=y(x)$ für $x=0, \mathbf{r}, 0,2,0,3, \ldots, r_{, 4}$ zehnstellig berechnet. Wir haben im Texte die Werte auf 7 Dezimalen abgekürzt. 
Zur numerischen Lösung von Randwertaufgaben bei Differentialgleichungen. 175 das Formelsystem III wesentlich genauere Werte als II, jene sind fast gleichwertig mit den aus IV ermittelten, obgleich nur zwei Gleichungen benutzt wurden. Auch bei Anwendung des Systems II sind die Febler der $y_{i}$ so klein, dass sie in graphischen Darstellungen wie Abb. 3 nicht sichtbar werden.

Tafel II.

\begin{tabular}{|c|c|c|c|c|}
\hline$i$ & $s$ & $x$ & $y$ appr. & $\dot{y}$ exakt \\
\hline - & $-\because$ & 0 & - & 1 \\
\hline I & $-\frac{1}{4}$ & 0,3 & I,, 000687 & 1,000675 \\
\hline 2 & 0 & 0,6 & I, $0108 \times 9$ & 1,010825 \\
\hline 3 & $+\frac{1}{4}$ & 0,9 & $I, 05529 x$ & I,055319 \\
\hline 一 & $+\frac{1}{2}$ & $I, 2$ & - & I, 179300 \\
\hline
\end{tabular}

Tafel III.

\begin{tabular}{|c|c|c|c|c|}
\hline $\begin{array}{l}\text { Formel- } \\
\text { system }\end{array}$ & $s$ & $x$ & $y$ appr. & $y$ exakt \\
\hline- & $-\frac{2}{2}$ & 0 & -- & 1 \\
\hline III & $-0,1864 \times 2$ & $0,37^{6} 305$ & $1, \infty 01696$ & $I, 00167^{2}$ \\
\hline II & $-\frac{1}{16}$ & 0,4 & 1,002017 & $I, 002134$ \\
\hline IJ & $+\frac{1}{16}$ & 0,8 & 1,024133 & $I, 0343^{8}+$ \\
\hline III & $+0,1864 \times 2$ & 0,823695 & $1,0386 \pi x$ & 1,038677 \\
\hline- & $+\frac{1}{2}$ & 1,2 & - & 1,179360 \\
\hline
\end{tabular}

25. Beispiel 2. Wir wollen noch eine Randwertaufgabe bei derselben Glei. chung (25) behandeln, aber in einem anderen Intervall. Die Randwerte seien jetzt (Abb. 3)

$$
x_{a}=\mathrm{I}, \mathrm{o}, \quad y_{a}=\mathrm{I}, 0848327, \quad x_{b}=\mathrm{I}, 4, \quad y_{b}=\mathrm{I}, 3427436
$$

und das System IV soll wieder angewandt werden. Zur Bestimmung des Wertes der gesuchten Funktion $y(x)$ in den drei Zwischenpunkten $x=1,1,1,2,1,3$ haben wir wieder drei simultane, unmittelbar aufzustellende Gleichungen. Nachdem die gegebenen Werte eingesetzt sind, lassen sich daraus die gresuchten Grössen $y_{1}, y_{2}, y_{3}$ leicht durch Iteration bestimmen. 
Als Ergebnis findet man die bis auf Abrundungsfehler der siebenstelligen Rechnung richtigen Werte der Tafel IV.

Bei der Bestimmung der in Rede stehenden Funktion $y=y(x)$ durch Lösung der Anfangswertaufgabe mit $y(0)=1, y^{\prime}(0)=0$ nimmt die Genauigkeit bei den Schritten von $x=1$,o an rasch ab, weil das betreffende Kurvenstück $A^{\prime} B^{\prime}$ in Abb. 3 immer steiler ansteigt. Bei den Randwertaufgaben dagegen, die von uns mit dem Formelsystem IV behandelt wurden, hat das im Intervall $A^{\prime} B^{\prime}$ erzielte Resultat sich als genauer erwiesen als dasjenige im Intervall $A B$.

\section{Tafel IV.}

\begin{tabular}{|c|c|c|c|c|}
\hline$i$ & 8 & $x$ & y appr. & $y$ exakt \\
\hline- & $-\frac{1}{2}$ & 1,0 & - & 1,0848327 \\
\hline $\mathbf{I}$ & $-\frac{1}{4}$ & $\mathbf{I}, \mathbf{r}$ & $I, 1252337$ & $1,125233^{8}$ \\
\hline 2 & o & 1,2 & $I, x 79299^{8}$ & I,1792999 \\
\hline 3 & $+\frac{1}{4}$ & 1,3 & $I, 2504 \times 27$ & $I, 2504129$ \\
\hline- & $+\frac{1}{2}$ & $\mathrm{~J}, 4$ & - & $I, 3427436$ \\
\hline
\end{tabular}

26. Beispiel 3. Auf eine lineare Randwertanfgabe der von uns botrachteten Form (6) kommt man u. a. in der Elastizitätslehre bei der Bestimmung der Amplitude $y(s)$ des elastischen Ausschlags eingespannter Saiten. ${ }^{1}$ Die festen Einspannungspunkte seien vorher mittels einer Transformation (3) auf $s= \pm \frac{1}{6}$ gebracht. Man erhält dann die Gleichung

$$
\frac{d^{2} y}{d s^{2}}=-\left(\mathrm{I}-p s^{2}\right) y-\mathrm{I}, \quad y\left( \pm \frac{1}{9}\right)=0
$$

Aus dieser wollen wir insbesondere den elastischen Ausschlag $y(0)$ in der Mitte zwischen den Einspannungsstellen bestimmen. Von den gegebenen Funktionen $\bar{g}(s)=-\left(\mathrm{I}-p s^{2}\right)$, wo $p \leqq 4$, und $h(s) \equiv-\mathrm{I}$, gibt die erste die im allgemeinen ungleichförmige, insbesondere sparabolische Massendichte an, wührend die zweite von der Einwirkung äusserer Kräfte herrührt. Wir betrachten die Fülle $p=0$, d. h. gleichförmige Massenverteilung, sodann den Fall $p=1$ und schliesslich den Grenzfall $p=4$, wo die Massendichte an den Enden der Saite auf Null herabsinkt.

1 Frank-Migks: Die Diferential- nnd Integralgleichungen der Mechanik nnd Physik, Bd. I Brannschweig 1925, S. 439 ff. 
Zur numerischen Lösung von Randwertaufgaben bei Differentialgleichungen. 177

Mit den gegebenen Ausdrücken von $\bar{y}$ und $h$ lassen sich unsere Formeln I, IV und $V$ bequem anwenden ${ }^{1}$.

Aus I erhalten wir mit $x_{a}=-\frac{1}{2}, x_{b}=+\frac{1}{2}, y_{a}=y_{b}=0, x=s$ unmittelbar einen Näherungswert $y_{1}=y(0)=0,1395$, der aber noch keine Abhängigkeit von $p$ zeigt.

Die Formelsysteme IV und V liefern infolge der Symmetrie je ein System von nur zwei Gleichungen, woraus wir die uns allein interessierende Unbekannte $y_{2}=y(0)$ leicht ermitteln können. Die Genauigkeit ist auch in dem Grenzfall $p=4$ beträchtlich, wie aus Tafel V hervorgeht.

\section{Tafel $V$.}

$$
y^{\prime \prime}(8)=-\left(1-p 8^{2}\right) y(8)-1, \quad y( \pm 1)=0
$$

Werte $y(0)$

\begin{tabular}{|c|l|l|l|}
\hline Formelsystem & $p=0$ & $p=\mathrm{I}$ & $p=4$ \\
\hline I & 0,1395 & 0,1395 & 0,1395 \\
IV & 0,13949 & 0,13908 & 0,13757 \\
V & 0,139494 & 0,139008 & 0,137575 \\
\hline Exakter Wert & 0,139494 & 0,139008 & 0,137574 \\
\hline
\end{tabular}

\section{Tafel VI.}

Beispiel 4.

Beispiel 5.

$$
\begin{array}{cc}
y^{\prime \prime}+y-2 \cos 8=0, & y^{\prime \prime}+y+2 \frac{\cosh 8}{\cosh \frac{1}{2}}-1=0, \\
y\left( \pm \frac{1}{2}\right)=0, & y( \pm 1)=0, \\
y=8 \sin 8-\frac{1}{2} \operatorname{tg} \frac{1}{2} \cos 8 . & y=1-\frac{\cosh 8}{\cosh \frac{1}{2}}
\end{array}
$$

\begin{tabular}{|c|c|c|c|c|c|c|}
\hline \multirow{2}{*}{ Formelsystem } & \multirow{2}{*}{$i$} & \multirow{2}{*}{8} & \multicolumn{2}{|c|}{ Beispiel 4} & \multicolumn{2}{|c|}{ Beispiel 5} \\
\hline & & & y appr. & $y$ exakt & $y$ appr. & $y$ exakt \\
\hline I & $\mathbf{I}$ & o & $-0,27337$ & $-0,273 \times 5$ & 0,12318 & $0,1\{33\}$ \\
\hline II & I, 2 & $\pm \frac{1}{6}$ & $-0,2477528$ & $-0,2417 \times 67$ & 0,1008409 & 0,2008357 \\
\hline III & 1,2 & $\pm 0,186_{4} 124$ & $-0,233^{8} 700$ & $-0,233^{869 x}$ & 0,0977283 & 0,0977283 \\
\hline
\end{tabular}

1 In der zitierten Arbeit des Verf. in Soc. Scient. Fenn., Comm. Phys.-Math. XI. 14. wird nntersucht, welche Genanigkeit erreicht werden kann, falls die Koeftizienten $g$ und $h$ einer Gleichung der Form (2) empirisch gegebene Funktionen wären. 
27. Es seien hier zwei gewissermassen willkürliche Beispiele 4 und 5 angeführt, die insbesondere zum Vergleich der Anwendung des Formelsystems II und des schärferen III dienen können. Wie die in Tafel VI zusammengestellten Ergebnisse zeigen, unterscheiden sich schon die mit dem einfacheren System II erhaltenen Werte so wenig von den exakten, dass es etwa beim Entwerfen einer Figur gleichgültig ist, welche ron ihnen benutzt werden. Die dabei erwünschten Ordinaten $y(0)$, können mit für zeichnerische $Z$ wecke ebenfalls genügender Genauigkeit aus der Formel I ermittelt werden.

Wir sehen aus diesen Beispielen, mit wie wenigen Punkten man in manchen Fällen den Verlauf der gesuchten Funktion festlegen kann. Auch die folgenden Beispiele können zur Erläuterung desselben Umstandes dienen.

28. Beispiel 6. In der schon genannten Arbeit des Verf. in Acta mathematica 54 ist die nichtlineare Randwertaufgabe behandelt

$$
y^{\prime \prime}(x)=\sin y(x)-\mathrm{I}, \quad y\left( \pm \frac{1}{2}\right)=0 .
$$

Es werden $d r e i$ Zwischenordinaten $y_{i}$ in Gauss-Lobattoscher Verteilung berechnet und mittels derselben die Näherungslösung aufgestellt

$$
y(x)=0,113200-0,443521 x^{y}-0,036750 x^{4}-0,001444 x^{6} .
$$

Bei Abrundung der Werte $y(x)$ auf fünf Dezimalen beträgt der Fehler höchstens eine Einheit der letzten Stelle.

Mit Rücksicht auf das in Nr. 18 Gesagte wissen wir, dass dieses Ergebnis auch mittels der Formeln IV hätte erzielt werden können.

29. Beispiel \%. Zum Schluss wählen wir ein etwas komplizierteres Beispiel bei einem nichtlinearen System von zwei Gleichungen, und zwar eines aus dem Gebiet der Himmelsmechanik. ${ }^{1}$

Ein Planet $P$ möge, von einem Zentralkörper $S$ angezogen, eine gewisse, die bekannten Punkte $A$ und $B$ verbindende Bahn beschreiben, in deben er sich $\mathrm{zu}$ den Zeiten $t_{a}=0$ und $t_{b}=12$ befindet, wenn für die Bewegung die Gleichungen gelten

$$
\left\{\begin{array}{lc}
\frac{d^{2} x}{d t^{2}}=-k^{2}(\mathrm{I}+m) \frac{x}{r^{8}}, & r^{2}=x^{2}+y^{2} \\
\frac{d^{2} y}{d t^{2}}=-k^{2}(1+m) \frac{y}{r^{3}}, & k^{2}(\mathrm{I}+m)=\frac{\pi^{2}}{324}
\end{array}\right.
$$

1 Dasselbe ist als Anfangswertproblem behandelt in der Arbeit des Verf.: Über die numerische Integration von Differentialgleichungen (Acta Soc. Scient. Fenn. L. N:0 I3 (1925)), S. 52-55. 
Zur numerischen Lösung von Randwertaufgaben bei Differentialgleichungen. 179

$$
x_{a}=\mathrm{I}, 8660254, \quad y_{a}=0, \quad x_{b}=\mathrm{I}, 1643915, \quad y_{b}=0,4772257 .
$$

$k^{2}$ ist die Gravitationskonstante und $m$ ist das Verhältnis der Massen des Planeten und des Zentralkörpers.

Unsere Aufgabe wird auf das Intervall $\left(-\frac{1}{2},+\frac{1}{2}\right)$ mit den Randwerten Null durch folgende Formeln zurückgeführt

$$
\left\{\begin{array}{l}
t=12 s+6 \\
x=\bar{x}+\mathrm{I}, 5152086-0,7016336 s \\
y=\bar{y}+0,2386129+0,4772257 s
\end{array}\right.
$$

Das zu lösende Gleichungssystem wird

$$
\left\{\begin{array}{l}
\frac{d^{2} \cdot \bar{x}}{d s^{2}}=-\frac{4 \pi^{2}}{9} \cdot \frac{x}{\gamma^{8}}, \\
\frac{d^{2} \bar{y}}{d s^{2}}=-\frac{4 \pi^{2}}{9} \cdot \frac{y}{r^{3}} .
\end{array}, \quad, \quad x^{2}=x^{2},\right.
$$

Unter Anwendung des Formelsystems II exhalten wir nach Einsetzung der gegebenen Randwerte $x_{1}, y_{1}, x_{2}, y_{2}$, fünfstellig

$$
\left\{\begin{array}{l}
x_{1}=1,63215-\frac{\pi^{3}}{729}\left[0,57437+21 \frac{x_{1}}{r_{1}^{3}}+12 \frac{x_{2}}{r_{1}^{3}}+0,58432\right], \\
x_{z}=1,39827-\frac{\pi^{2}}{729}\left[0,28719+12 \frac{x_{1}}{r_{1}^{3}}+21 \frac{x_{2}}{r_{2}^{3}}+1,16864\right] \\
y_{1}=0,15908-\frac{\pi^{2}}{729}\left[0+21 \frac{y_{1}}{r_{1}^{3}}+12 \frac{y_{z}}{r_{3}^{3}}+0,23948\right], \\
y_{z}=0,31815-\frac{\pi^{2}}{729}\left[0+12 \frac{y_{1}}{r_{2}^{3}}+21 \frac{y_{3}}{r_{2}^{3}}+0,47896\right] .
\end{array}\right.
$$

Um aus diesen Gleichungen die Werte der vier Unbekannten $x_{1}, y_{1}, x_{z}, y_{2}$ durch sukzessive Approximation zu ermitteln, gehen wir mit dem runden Wert $r^{3}=5$ in (3I) ein. Dann ergeben sich leicht Näherungswerte für die gesuchten Grössen. Diese gestatten, einen besseren Wert für $r^{3}$ zu berechnen, und dann kann man die Rechnung fortsetzen. In der dritten Approximation erhält man $x$ - und $y$-Werte, die als endgültig betrachtet werden können. Sie sind in Tafel VII angegeben und zeigen erst in der vierten Dezimalstelle Abweichungen von den exakten Werten. Die letzteren findet man aus den Gleichungen

$$
x=\frac{\sqrt{3}}{2}+\cos u, \quad y=\frac{1}{2} \sin u, \quad u+\frac{\sqrt{3}}{2} \sin u=\frac{\pi}{18} t .
$$


Tafel VII.

\begin{tabular}{|c|c|c|c|c|c|c|}
\hline Formelsystem & $t$ & 8 & $x$ appri. & $x$ exakt & $y$ appr. & $y$ exakt \\
\hline- & 0 & $-\frac{1}{2}$ & - & 1,8660 & - & 0,0000 \\
\hline II & 4 & $-\frac{1}{6}$ & $I, 794^{8}$ & I,7953 & 0,1848 & $0, \times 847$ \\
\hline I & 6 & 0 & 1,706 & $I, 705$ & 0,274 & $0.27^{2}$ \\
\hline II & 8 & +6 & 1,5735 & $I, 573^{8}$ & 0,3535 & $0,353^{2}$ \\
\hline- & 12 & +1 & - & $I, x 644$ & - & $0,477^{2}$ \\
\hline
\end{tabular}

Das betrachtete Stück der Planetenbahn geht vom Aphel $A$ aus (Abb. 4), von welchem Umstand wir aber keinen Gebrauch gemacht haben.

Wir wollen noch unsere Formel I auf dasselbe Beispiel anwenden. Hierbei bleiben (29) und (30) bestehen. Statt (31) erhalten wir jetzt das Gleichungssystem

$$
\left\{\begin{array}{l}
x_{1}=1,5152-\frac{\pi^{2}}{216}\left[0,2872+10 \frac{x_{1}}{r_{1}^{3}}+0,5843\right], \\
y_{1}=0,2386-\frac{\pi^{2}}{216}\left[0+10 \frac{y_{1}}{r_{1}^{3}}+0,2395\right],
\end{array}\right.
$$

wo also $x_{1}$ und $y_{1}$ die Koordinaten zur Zeit $t_{1}=6$ bedeuten.

Bereits in der zweiten Approximation erhält man in der beschriebenen Weise die in Tafel VII angegebenen, endgültigen Werte. In Abb. 4 ist der Deutlichkeit wegen das betreffende Bahnstück auf der entgegengesetzten Seite des Aphels als das vorher betrachtete dargestellt.

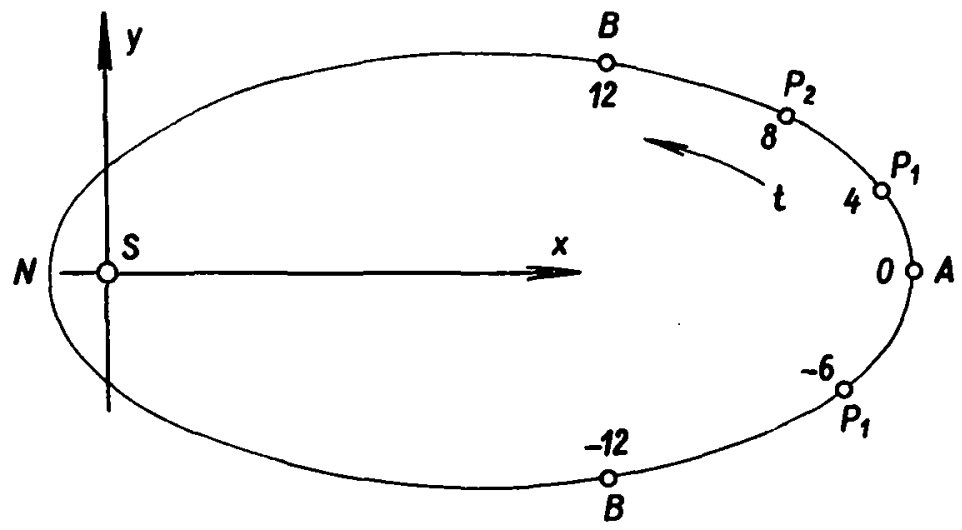

Abl). 4 . 
Zur numerischen Lösung von Randwertaufgaben bei Differentialgleichungen. 181

Das obige Beispiel dürfte genügen, um die Anwendbarkeit unserer Methoden auf die Integration von Bewegungsgleichungen zu zeigen. Die Methoden haben natürlich auch ihre Begrenzung: Wollte man z. B. das Baḅnstück zwischen dem Punkt $B$ und dem Perihel $N$ durch Lösung einer Randwertaufgabe ermitteln, so würde man finden, dass es sich hierbei um einen viel zu kühnen Sprung handelt. In der Tat würde in dem Ausdruck des Fehlergliedes eine hohe Potenz der kleinen Grösse $\overline{S N}$ auftreten und der Fehler infolgedessen einen grossen Betrag erreichen. Die in Nr. 8 und I I genannte Bedingung wäre nicht erfüllt. Bei einer weniger exzentrischen Bahn würde indessen auch dieses Problem befriedigend gelöst werden können.

\section{§ 7. Schlussbetrachtung.}

30. Wir haben gewissermassen die bekannte Gausssche Integrationsmethode auf Randwertaufgaben übertragen durch unseren Versuch, mit wenigen, zweckmässig gewählten Ordinaten auszukommen. ${ }^{1}$

Bei einer Vergrösserung der Anzahl der Zwischenordinaten wächst zwar die Genauigkeit des Resultats aber auch die zu leistende Rechenarbeit. Bei langem Abstand der gegebenen Punkte sowie bei irgendwelchen Singularitäten ist überhaupt kein gutes Resultat zu erwarten.

Die behandelten Beispiele geben Aufschluss darüber, bei welcher Art von Aufgaben unsere Formelsysteme sich als erfolgreich erweisen. In vielen Fällen genügen schon die einfacheren; das System VI mit 4 Zwischenordinaten haben wir überhaupt nicht für unsere eigentlichen Beispiele nötig gehabt. Im allgemeinen dürfte das System IV und in schwierigeren Fällen VI das geeignetste sein, jedoch sind nach der Ansicht des Verf. auch die mit nicht äquidistanten Ordinaten arbeitenden Systeme III und $\nabla$ von Bedeutung.

Bei der Anwendung der Systeme II oder III hat man ein nur aus 2 simultanen Gleichungen bestehendes System zu lösen. In diesem Fall ist natürlich, wie auch sonst, die Auflösung durch Iteration das Nächstliegende. Auf graphischem Wege lassen sich leicht brauchbare Ausgangswerte ermitteln, denn solche findet man durch Zeichnen von zwei Kurvenstücken und Aufsuchen ihres Schnittpunktes.

Schon unser einfachstes Verfahren I gestattet in manchen Fällen, die Randwertaufgabe mit einer Genauigkeit zu lösen, die etwa der zeichnerisch erreichbaren entspricht.

1 Die entsprechende Ubertragang anf Integralgleichingen findet man in drei Arbeiten des Verf., nämlich Soc. Scient. Fenn., Comm. Phys.-Math. IV. I 5. (1928) und in den beiden ersten der in Nr. 2, Fussnote 2 genanoten Veröffentlichungen. 


\section{Formelzusammenstellung.}

Bei der Randwertaufgabe

$$
\frac{d^{2} y}{d x^{2}}=f(x, y), \quad y\left(x_{c}\right)=y_{a}, \quad y\left(x_{b}\right)=y
$$

können Näherungswerte der Zwischenordinaten $y_{1}, y_{2}, \ldots$ aus irgendeinem der Gleichungssysteme I-VI berechnet werden. Im Falle einer linearen Differentialgleichung, $f(x, y)=g(x) y+h(x)$, werden die Gleichungssysteme linear. Die Bedeutung der Korrektionsglieder $\Delta y_{i}$ ist in Nr. 12 angegeben.

I. Eine Zwischenordinate $y_{1}$, äquidistant.

(vgl. Nr. 17)

$$
s=0, \quad x_{1}=\frac{1}{2}\left(x_{a}+x_{b}\right),
$$

$$
\begin{gathered}
y_{1}=\underline{1}\left(y_{a}+y_{b}\right)-\frac{\left(x_{b}-x_{a}\right)^{2}}{96}\left[f\left(x_{a}, y_{a}\right)+\operatorname{Iof}\left(x_{1}, y_{1}\right)+f\left(x_{b}, y_{b}\right)\right], \\
\Delta y_{1}=-0,000781 \cdot c_{1}+\cdots
\end{gathered}
$$

II. Zwei Zwischenordinaten $y_{1}, y_{2}$, äquidistant.

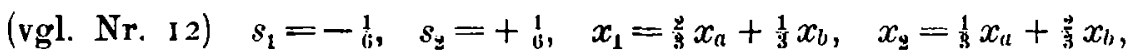

$$
\left\{\begin{array}{c}
y_{1}=\frac{2}{3} y_{a}+\frac{1}{3} y_{b}-\frac{\left(x_{b}-x_{a}\right)^{2}}{324}\left[2 f\left(x_{a}, y_{a}\right)+2 \text { I } f\left(x_{1}, y_{1}\right)+12 f\left(x_{9}, y_{2}\right)+f\left(x_{b}, y_{b}\right)\right], \\
y_{8}=\frac{1}{3} y_{\alpha}+\frac{2}{3} y_{b}-\frac{\left(x_{b}-x_{a}\right)^{2}}{324}\left[f\left(x_{a}, y_{a}\right)+12 f\left(x_{1}, y_{1}\right)+2 \text { I } f\left(x_{2}, y_{2}\right)+2 f\left(x_{b}, y_{b}\right)\right], \\
\Delta y_{1}=\Delta y_{z}=-0,0001371 c_{1}+\cdots .
\end{array}\right.
$$

III. Zwei Zwischenordinaten $y_{1}, y_{2}$, nicht äquidistant.

(vgl. Nr. 13)

$$
s_{1}=-\sqrt{\frac{\mathrm{II}-\sqrt{112}}{\mathrm{I} 2}}=-0,1864124=-s_{2},
$$

$$
x_{1}=0,6864124 x_{a}+0,3135876 x_{b}, \quad x_{2}=0,3135876 x_{a}+0,6864124 x_{b} \text {. }
$$

$$
\left\{\begin{array}{c}
y_{1}=0,6864124 y_{a}+0,3135876 y_{b}- \\
\left(x_{b}-x_{a}\right)^{2}\left[0,0047517 f\left(x_{a}, y_{a}\right)+0,0643500 f\left(x_{1}, y_{1}\right)+0,0369208 f\left(x_{2}, y_{z}\right)+0,0016028 f\left(x_{b}, y_{b}\right)\right], \\
y_{2}=0,3135876 y_{a}+0,6864124 y_{b}- \\
\left(x_{b}-x_{a}\right)^{2}\left[0,0016028 f\left(x_{a}, y_{a}\right)+0,0369208 f\left(x_{1}, y_{1}\right)+0,0643500 f\left(x_{2}, y_{2}\right)+0,0047517 f\left(x_{b}, y_{b}\right)\right],
\end{array}\right.
$$

$$
\Delta y_{1}=-\Delta y_{z}=+0,0000354 \cdot c_{3}+\cdots
$$


Zur numerischen Lösung von Randwertaufgaben bei Differentialgleichungen. 183

IV. Drei Zwischenordinaten $y_{1}, y_{2}, y_{s}$, äquidistant.

(vgl. Nr. I4)

$$
s_{1}=-\frac{1}{4}, \quad s_{2}=0, \quad s_{3}=+1,
$$

$$
x_{1}=\frac{3}{4} x_{a}+\frac{1}{4} x_{b}, \quad x_{z}=\frac{1}{2}\left(x_{a}+x_{b}\right), \quad x_{3}=\frac{1}{4} x_{a}+\frac{3}{1} x_{b},
$$

$$
\left\{\begin{aligned}
y_{1}=\frac{3}{4} y_{a}+\frac{1}{4} y_{b}- & \\
& \frac{\left(x_{b}-x_{a}\right)^{2}}{7680}\left[27 f\left(x_{a}, y_{a}\right)+332 f\left(x_{1}, y_{1}\right)+222 f^{\prime}\left(x_{2}, y_{2}\right)+132 f\left(x_{3}, y_{3}\right)+7 f\left(x_{b}, y_{b}\right)\right], \\
y_{2}=\frac{1}{2} y_{a}+\frac{1}{2} y_{b}- & \\
& \frac{\left(x_{b}-x_{a}\right)^{2}}{480}\left[f\left(x_{a}, y_{n}\right)+16 f\left(x_{1}, y_{1}\right)+26 f\left(x_{2}, y_{2}\right)+16 f\left(x_{3}, y_{3}\right)+f\left(x_{b}, y_{b}\right)\right], \\
y_{3}=\frac{1}{4} y_{a}+\frac{3}{1} y_{b}- & \frac{\left(x_{b}-x_{a}\right)^{2}}{7680}\left[7 f\left(x_{n}, y_{n}\right)+132 f\left(x_{1}, y_{1}\right)+222 f\left(x_{2}, y_{z}\right)+332 f\left(x_{3}, y_{3}\right)+27 f\left(x_{b}, y_{b}\right)\right] .
\end{aligned}\right.
$$$$
\Delta y_{1}=-\Delta y_{3}=0,0000152 \cdot c_{5}+\cdot, \quad \Delta y_{2}=-0,0000116 \cdot c_{6}+\cdots
$$

V. Drei Zwischenordinaten $y_{1}, y_{2}, y_{3}$, nicht äquidistant.

(vgl. Nr. I 5) $s_{1}=-\sqrt{\frac{19-\sqrt{240}}{44}}=-0,2823630=-s_{3}, \quad s_{2}=0$.

$$
x_{1}=0,7823630 x_{a}+0,2176370 x_{l}, \quad x_{2}=\frac{1}{2}\left(x_{a}+x_{b}\right), \quad x_{3}=0,2176370 x_{a}+0,7823630 x_{b} .
$$

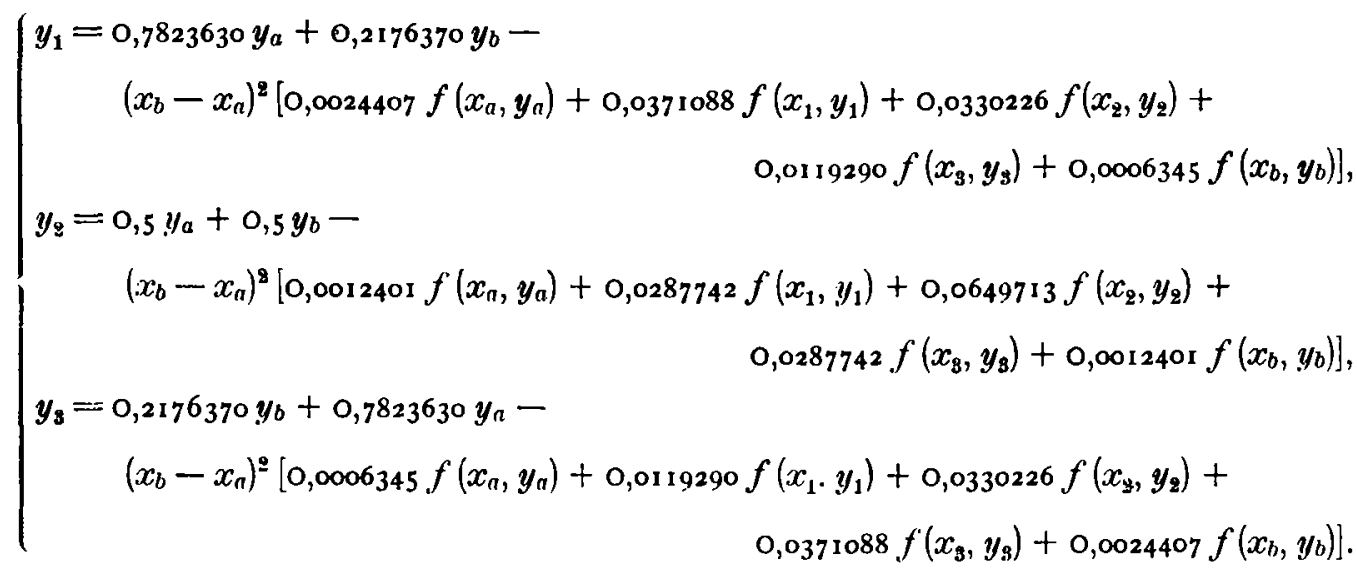

$$
\begin{aligned}
& \Delta y_{1}=\Delta y_{3}=-0,0000039 \cdot c_{6}+\cdots, \quad \Delta y_{z}=0,0000019 \cdot c_{6}+\cdots
\end{aligned}
$$


VI. Vier Zwischenordinaten $y_{1}, y_{2}, y_{3}, y_{4}$, äquidistant.

(vgl. Nr. I6) $s_{1} \neq-0,3, \quad s_{2}=-0, \mathrm{i}, \quad s_{\mathrm{g}}=+0, \mathrm{r}, \quad s_{4}=+0,3$

$$
\begin{aligned}
& x_{1}=0,8 x_{n}+0,2 x_{b}, \quad x_{3}=0,6 x_{a}+0,4 x_{b}, \quad x_{3}=0,4 x_{a}+0,6 x_{b}, \quad x_{4}=0,2 x_{n}+0,8 x_{b} \\
& \left\{\begin{array}{l}
y_{1}=0,8 y_{a}+0,2 y_{b}- \\
\quad \frac{\left(x_{b}-x_{n}\right)^{2}}{3000}\left[7 f\left(x_{n}, y_{a}\right)+90 f\left(x_{1}, y_{1}\right)+66 f\left(x_{2}, y_{2}\right)+52 f\left(x_{3}, y_{3}\right)+\right.
\end{array}\right. \\
& y_{z}=0,6 y_{a}+0,4 y_{b}- \\
& \left.23 f\left(x_{4}, y_{4}\right)+2 f\left(x_{b}, y_{b}\right)\right] \text {, } \\
& \frac{\left(x_{b}-x_{a}\right)^{2}}{6000}\left[\operatorname{Iof}\left(x_{11}, y_{a}\right)+151 f\left(x_{1}, y_{1}\right)+260 f\left(x_{2}, y_{2}\right)+194 f\left(x_{3}, y_{3}\right)+\right. \\
& y_{3}=0,4 y_{a}+0,6 y_{b}- \\
& \left.98 f\left(x_{k}, y_{4}\right)+7 f\left(x_{b}, y_{b}\right)\right] \\
& \frac{\left(x_{b}-x_{a}\right)^{2}}{6000}\left[7 f\left(x_{a}, y_{a}\right)+98 f\left(x_{1}, y_{1}\right)+194 f\left(x_{2}, y_{2}\right)+260 f\left(x_{3}, y_{3}\right)+\right. \\
& y_{4}=0,2 y_{a}+0,8 y_{b}- \\
& \text { I } \left.5 \text { I } f\left(x_{4}, y_{4}\right)+\operatorname{IO} f\left(x_{b}, y_{b}\right)\right] \text {, } \\
& \frac{\left(x_{b}-x_{a}\right)^{2}}{3000}\left[2 f\left(x_{a}, x_{a}\right)+23 f\left(x_{1}, y_{1}\right)+52 f\left(x_{2}, y_{2}\right)+66 f\left(x_{3}, y_{3}\right)+\right. \\
& \left.90 f\left(x_{1}, y_{4}\right)+7 f\left(x_{b}, y_{b}\right)\right] \text {. } \\
& \Delta y_{1}=\Delta y_{1}=-0,0000058 \cdot c_{6}+\cdots, \\
& \Delta y_{2}=\Delta y_{8}=-0,0000048 \cdot c_{6}+\cdots \text {. }
\end{aligned}
$$

